\title{
Molecular Dynamics Simulations of Enantiomeric Separations as an Interfacial Process in HPLC
}

\author{
Cynthia Jameson ${ }^{1}$, Xiaoyu Wang ${ }^{2}$, and SOHAIL MURAD ${ }^{3}$ \\ ${ }^{1}$ University of Illinois at Chicago \\ ${ }^{2}$ Illinois Institute of Technology \\ ${ }^{3}$ Illinois Institute of Technology Armour College of Engineering
}

June 27, 2020

\begin{abstract}
Since chromatographic separation is a dynamic process, with the interactions between the drug and the chiral stationary phase mediated by the solvent, no single interacting structure, such as could be found by minimizing the energy, could possibly describe and account for the ratio of residence times in the chromatographic column for the enantiomeric pair. We describe the use of explicit-solvent fully atomistic molecular dynamics simulations, permitting all the interactions between the atoms constituting the chiral stationary phase, solvent molecules and the drug molecule. This allows us to better understand the molecular dynamic chiral recognition that provides the discrimination which results in the separation of enantiomers by high performance liquid chromatography. It also provides a means of predicting, for a given set of conditions, which enantiomer elutes first and an estimate of the expected separation factor. In this review we consider the use of molecular dynamics towards this understanding and prediction.
\end{abstract}

\section{Hosted file}

Jameson -AICHE -Gubbins.pdf available at https://authorea.com/users/337462/articles/463054molecular-dynamics-simulations-of-enantiomeric-separations-as-an-interfacial-process-in$\mathrm{hplc}$ 